\title{
miR-642 serves as a tumor suppressor in hepatocellular carcinoma by regulating SEMA4C and p38 MAPK signaling pathway
}

\author{
ZAIJUN YU ${ }^{1 *}$, YUEHE DU $^{2 *}$, HONGYING LI $^{1}$, JICHAO HUANG $^{1}$, DEQING JIANG ${ }^{1}$, JILONG FAN $^{1}$, \\ YUELAN SHEN $^{1}$, LINGLING ZHANG ${ }^{1}$, XIUJUAN YU ${ }^{1}$, NA XU $^{1}$ and QUNGANG KE ${ }^{1}$ \\ ${ }^{1}$ Department of Hepatobiliary Surgery, The Second People's Hospital of Lianyungang, Lianyungang, \\ Jiangsu 222006; ${ }^{2}$ Department of Emergency Office, Center for Disease Control and \\ Prevention of Lianyungang, Lianyungang, Jiangsu 222003, P.R. China
}

Received October 1, 2019; Accepted June 11, 2020

DOI: 10.3892/ol.2020.11935

\begin{abstract}
Hepatocellular carcinoma (HCC) is a malignant tumor with high incidence and high risk. Study of the role and mechanism of miRNAs are a hot spot of research providing new treatment ideas in malignant tumors. The effect of miR-642a on HCC progression and the underlying molecular mechanism were investigated. Expression of miR-642a and SEMA4C was measured by western blot analysis and RT-PCR. miR-642a expression was elevated while SEMA4C expression was attenuated in HCC tissues and cells. Results of luciferase reporter and western blot analyses show that miR-642a modulated SEMA4C expression by binding to its 3'UTR. Moreover, miR-642a negatively regulated SEMA4C expression. HCC cell migration and invasion was tested by Transwell assays. The findings revealed that the number of migrated and invaded cells were reduced by miR-642a mimic and raised by miR-642a inhibitor, indicating that miR-642a showed a suppression effect on HCC cell migration and invasion. Additionally, the migration and invasion of HCC cells were inhibited by SEMA4C siRNA, and SEMA4C reversed miR-642a effect on HCC migration and invasion. Furthermore, p38 MAPK signaling pathway was proven to be inhibited by miR-642a mimic, whereas facilitated by miR-642a inhibitor and SEMA4C siRNA could overturn the promotion effect of miR-642a inhibitor. Briefly, miR-642a targeted SEMA4C to repress HCC cell migration and invasion through $\mathrm{p} 38$ MAPK signaling pathway providing a new strategy for treatment of HCC patients.
\end{abstract}

Correspondence to: Dr Qungang Ke, Department of Hepatobiliary Surgery, The Second People's Hospital of Lianyungang, 41 Hailian East Road, Haizhou, Lianyungang, Jiangsu 222006, P.R. China E-mail: zhulzqk69913@163.com

*Contributed equally

Key words: miR-642a, hepatocellular carcinoma, SEMA4C, migration, invasion

\section{Introduction}

Liver cancer can be divided into two major categories: primary and secondary liver cancer. Primary liver cancer originates from the epithelial or mesenchymal tissue of the liver, it is a malignant tumor with high incidence and great harm in China $(1,2)$. Hepatocellular carcinoma (HCC) is a type of primary liver cancer, and it is one of the most common malignancies worldwide (3). Most patients with $\mathrm{HCC}$ are diagnosed with advanced disease, resulting in low survival and poor prognosis due to lack of effective treatment $(4,5)$. Therefore, exploring the pathogenesis of HCC and discovery of an effective treatment strategy is very urgent.

Mounting evidence displays that the dysregulation of miRNAs is involved in a variety of cancers and modulate tumor development via targeting their mRNA, including HCC $(6,7)$. For example, miR-451a was shown to have inhibitory effect on HCC tumorigenesis through targeting LPIN1 (8). Wu et al (9) reported that miR-3650 inhibited HCC metastasis via targeting NFASC. However, miR-1307 enhanced HCC metastasis and tumor growth by inhibiting DAB2 (10). Upregulation of miR-494 promoted the development of HCC by targeting SIRT3 through TGF- $\beta /$ SMAD signaling pathway (11). Importantly, previous studies have shown that miR-642a was downregulated in HCC and associated with clinicopathological information (12). However, the role and precise molecular mechanisms of miR-642a in HCC cell migration and invasion has not been reported yet.

SEMA4C, a member of the semaphorin family, is upregulated in various cancers and take part in tumor growth, metastasis, and apoptosis through several signal transduction pathways, which is closely related to the progression and development of cancers. For example, Gurrapu et al (13) found that SEMA4C was overexpressed in breast cancer and served as an oncogene in modulating cell growth. Moreover, SEMA4C was significantly increased in tumor-associated lymphatic endothelial cells and showed facilitating effects on lymphatic metastasis (14). Furthermore, SEMA4C promoted malignant glioma cell invasion and was correlated with glioma poor survival (15). In addition, SEMA4C was proven to be 
upregulated in $\mathrm{HCC}$ and regulated $\mathrm{HCC}$ cell invasion and migration (16). However, whether SEMA4C affected the role of miR-642a in HCC metastasis is not very clear.

p38 MAPK signaling pathway is involved in cell growth, differentiation, inflammatory response and other important cellular physiological/pathological processes. Previous studies have provided evidence that SEMA4C might be an activator for p38 MAPK pathway (17). Here, we investigated whether p38 MAPK pathway participated in HCC development regulated by miR-642a/SEMA4C axis.

In the present study, the role and potential mechanism of miR-642a in HCC metastasis were investigated. The findings show that miR-642a was decreased in HCC and re-expression of miR-642a curbed HCC cell invasion and migration. On the contrary, SEMA4C showed a facilitating effect on HCC metastasis. SEMA4C was involved in HCC progression modulated by miR-642a, which provides a potential target for treatment of HCC patients.

\section{Patients and methods}

HCC specimens. Sixty pairs of HCC and paracancerous tissues were obtained from patients who underwent hepatectomy at The Second People's Hospital of Lianyungang (Lianyungang, China) from August 2013 to September 2018. The patients received no radiotherapy or chemotherapy before surgery. The study was approved by the Ethics Committee of The Second People's Hospital of Lianyungang, and an informed consent was signed by each patient. The fresh HCC tissues were immediately frozen at $-80^{\circ} \mathrm{C}$ for RT-PCR analysis.

Cell lines and cell culture. HCC cells (Huh7, HCCLM3) and normal liver epithelial cells THLE-3 were cultivated in RPMI-1640 medium containing 20\% FBS and antibiotics at $37^{\circ} \mathrm{C}$ under the atmosphere of $5 \% \mathrm{CO}_{2}$. The cells were obtained from Shanghai Institute of Chinese Academy of Sciences (Shanghai, China).

Cell transfection. Huh7 cells were added to 24-well plates containing RPMI-1640 medium. The transfection was performed for $48 \mathrm{~h}$ using Lipofectamine 3000 reagent (Invitrogen; Thermo Fisher Scientific, Inc.) according to the manufacturer's instructions. The miR-642a mimic, inhibitor and negative control used in this study were purchased from GenePharma. The miR-642a mimic or inhibitor was transfected into Huh7 cells to overexpress or silence miR-642a. SEMA4C siRNA and con siRNA were synthesized by GenePharma, and they were used to downregulate SEMA4C or utilize as a control. The miR-642a mimics sequence was: 5'-AGGACAGGGGAGGATTGCAACG-3'. The sequences of miR-642a inhibitor and scramble oligonucleotides were as follows: 5'-CACAGACGGAGGCCAGGGGAGA-3'; and 5'-CCGAAACCUCGGUUGAUUGCGG-3'. Con-siRnA, 5'-UUCUCCGAACGUGUCACGUTT-3'; SEMA4C-siRNA, 5'-CCUAUGCCUUCCAGCCCAAdTdT-3'.

RNA extraction and RT-PCR. TRIzol reagent (Invitrogen; Thermo Fisher Scientific, Inc.) was used to extract total RNA from HCC cells and tissues. The mRNA expression was quantified by Platinum ${ }^{\mathrm{TM}}$ Taq DNA polymerase. The sequences of the primers were as follows: miR-642a-F: 5'-ATACAAAGCC TAAGATGAG-3', miR-642a-R: 5'-GAGCAAGCTCCTAT TCC-3'; SEMA4C-F: 5'-ACCTTGTGCCGCGTAAGACAG-3', SEMA4C-R: 5'-CGTCAGCGTCAGTGTCAGGAA-3'; U6-F: 5'-CTCGCTTCGGCAGCACATATACT-3', U6-R: 5'-ACG CTTCACGAATTTGCGTGTC-3'; GAPDH-F: 5'-GATCATT GCTCCTCCTGAGC-3', GAPDH-R: 5'-ACTCCTGCTTGCT GATCCAC-3'. U6 and GAPDH were used as internal controls. Relative expression of miR-642a and SEMA4C was measured by the $2^{-\Delta \Delta C T}$ method.

Western blot analysis. Lysis buffer was used to extract proteins from $\mathrm{HCC}$ tissues or cells. After centrifugation at $12,000 \times \mathrm{g}, 4^{\circ} \mathrm{C}$ for $30 \mathrm{~min}$, the supernatant of tissues or cells was measured by BCA kit. Protein specimens $(50 \mu \mathrm{g})$ were added onto SDS-PAGE and electrophoresed at $60 \mathrm{~V}$. Proteins were then transferred to nitrocellulose filter membranes (NC). Subsequently, the membranes were blocked with skim milk (5-10\%) at $37^{\circ} \mathrm{C}$ for $1 \mathrm{~h}$ and then incubated with the primary antibodies against SEMA4C (1:500; sc-136445; Santa Cruz Biotechnology, Inc.), p38 (1:1000, ab170099; Abcam), p-p38 (1:1000, ab47363; Abcam), MAPK (1:1000, ab185145; Abcam), p-MAPK (1:2000, 4370; Cell Signaling Technology, Inc.,) at $4^{\circ} \mathrm{C}$ overnight. Then, the membranes were incubated with horseradish peroxidase-labeled secondary antibody (1:5000; Santa Cruz Biotechnology) for $1 \mathrm{~h}$ at $37^{\circ} \mathrm{C}$. GAPDH (1:2000; ab181602; Abcam) was used as the loading control. Finally, enhanced chemiluminescence kit (ECL; EMD Millipore) was used to detect the signals.

Transwell assay. Transwell assays were used to measure cell migration and invasion. For migration assay, the top and the lower chambers were separated by Transwell chamber with $8-\mu \mathrm{m}$ pore size polycarbonic membrane (Costar) in 6 -well plates. Huh7 cells $\left(5 \times 10^{5} /\right.$ well $)$ with different transfection were added into the top chambers and the lower chambers were fixed with DMEM containing 10\% FBS. Then, they were cultured for $24 \mathrm{~h}$ at $37^{\circ} \mathrm{C}$. When the cells migrated into the lower chambers, the cells were fixed using $100 \%$ methanol, stained with $0.1 \%$ crystal violet, photographed with an inverted microscope (Nikon 80i; Olympus) and counted with image software. For invasion assay, except the top chamber with the filter coated with Matrigel, it was the same as for the cell migration assay.

Luciferase reporter assay. The pEZX-MT06 vector containing the full length of SEMA4C 3'-UTR was purchased from GeneCopoeia Inc. Lipofectamine 2000 (11668027; Invitrogen; Thermo Fisher Scientific, Inc.) and used to perform transfection with miR-642a mimic and luciferase reporter vector containing wild-type (WT) or mutated (MuT) 3'UTR of SEMA4C into Huh7 cells. The Dual Luciferase Reporter system (E1910; Promega) was carried out to measure the luciferase activity of Huh7 cells treated with different transfection.

Statistical analysis. All the independent experiments were repeated at least three times and results are shown as mean \pm SD. Statistical analysis and graph presentations were performed, respectively, by SPSS v.19.0 software (SPSS, Inc.) and GraphPad Prism 6 software (GraphPad Inc.). The 
A

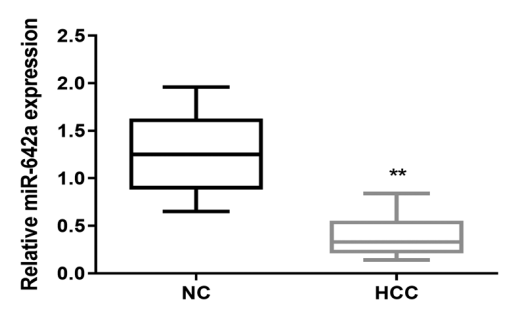

C

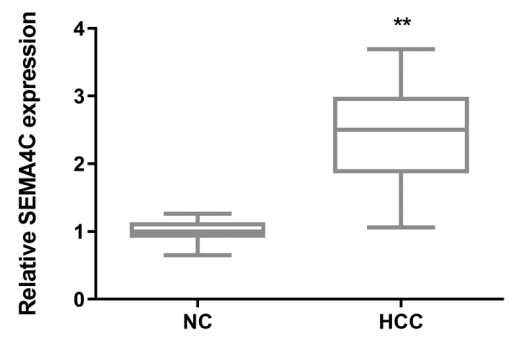

E

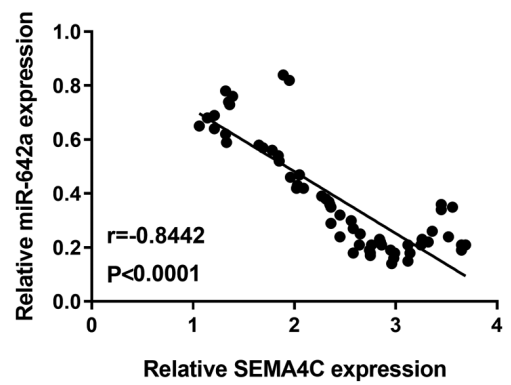

B

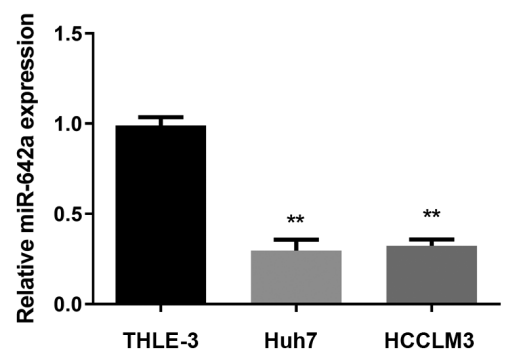

D

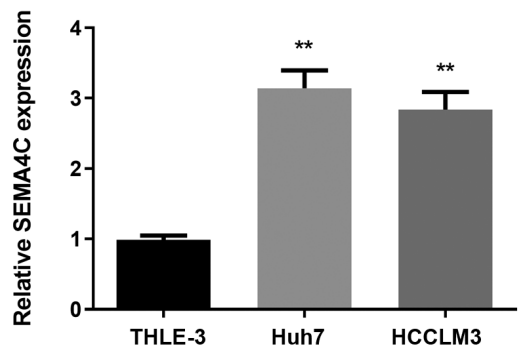

Figure 1. miR-642a is decreased and SEMA4C is increased in HCC. (A) Comparison of miR-642a expression in HCC tumor tissues and normal tissues $(\mathrm{n}=60)$. (B) Comparison of miR-642a expression in HCC cell lines (Huh7, HCCLM3) and normal cells (THLE-3). (C) Comparison of SEMA4C expression in HCC tumor tissues and normal tissues $(n=60)$. (D) Comparison of SEMA4C expression in HCC cell lines (Huh7, HCCLM3) and normal cells (THLE-3). (E) Detection of the correlation between miR-642a and SEMA4C expression $(\mathrm{r}=-0.8442, \mathrm{P}<0.001) .{ }^{* *} \mathrm{P}<0.01$. HCC, hepatocellular carcinoma.

differences between groups were compared using Student's t-test or one-way analysis of variance (ANOVA) followed by Tukey's post hoc test. Statistically significant difference was considered as $\mathrm{P}<0.05$.

\section{Results}

miR-642a expression is decreased and SEMA4C is increased in HCC. To investigate the biological functions of miR-642a and SEMA4C on HCC development, we first tested the expressional level of miR-642a and SEMA4C in HCC tissues and cells. RT-PCR results displayed that miR-642 expression was reduced in HCC tissues and cells compared with normal controls (Fig. 1A and B). However, SEMA4C showed the opposite expression in HCC tissues and cells (Fig. 1C and D). Then, the relationship between miR-642a and SEMA4C was measured in HCC tissues and the results revealed that they were negatively correlated (Fig. 1E).

SEMA4C serves as a target of miR-642a. Based on the above results, we needed to clarify whether SEMA4C acted as a candidate target of miR-642a in HCC. For verifying this hypothesis, PITA database was first applied to determine the binding sites of SEMA4C with miR-642a. As presented in Fig. 2A, they have the binding sites. Then, dual-luciferase reporter assay was applied to further confirm whether SEMA4C was the direct target of miR-642a in HCC cells. The findings revealed that the luciferase activity in Huh7 cells co-transfected with miR-642a mimic and SEMA4C-3'-UTR-WT was significantly decreased, while increased in Huh7 cells co-transfected with miR-642a inhibitor and SEMA4C-3'-UTR-WT. However, there was no significant difference in SEMA4C-3'-UTR-MUT (Fig. 2B). Next, we measured whether miR-642a regulated SEMA4C expression in Huh7 cells. Results of Western blot and RT-PCR analyses showed that re-expression of miR-642 was reduced, while silence of miR-642 elevated SEMA4C expression (Fig. 2C and D). The conclusion drawn from the above results suggested that miR-642a regulated SEMA4C expression by binding to its 3'UTR in HCC.

miR-642a represses HCC malignancy. The biological functions of miR-642a on HCC malignancy was measured. The 
A

SEMA4C 3'UTR-WT 5'UUUUCAGgGUGGgGgAGgGAG 3 ' | | |||||| hsa-miR-642a 3'GUUCUGUGUAAACCUCUCCCUG 5'

SEMA4C 3'UTR-MuT 5'UUUUCAGGGUGGGGGCUCGCAG 3'

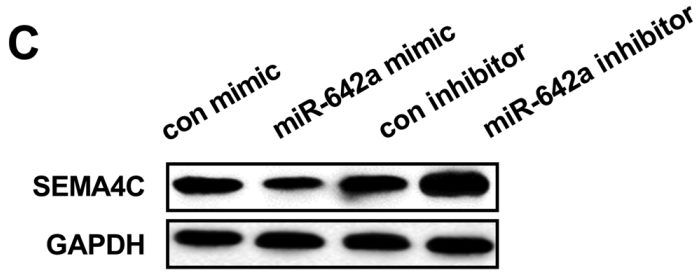

D

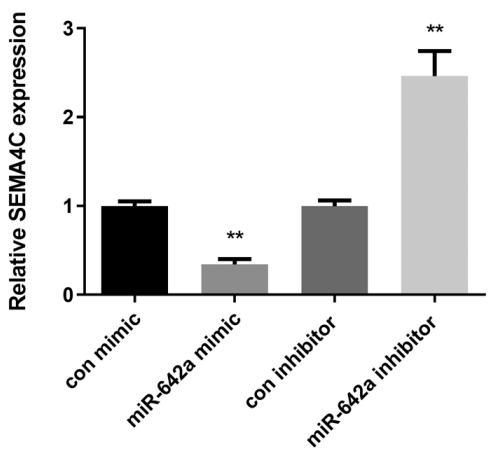

B
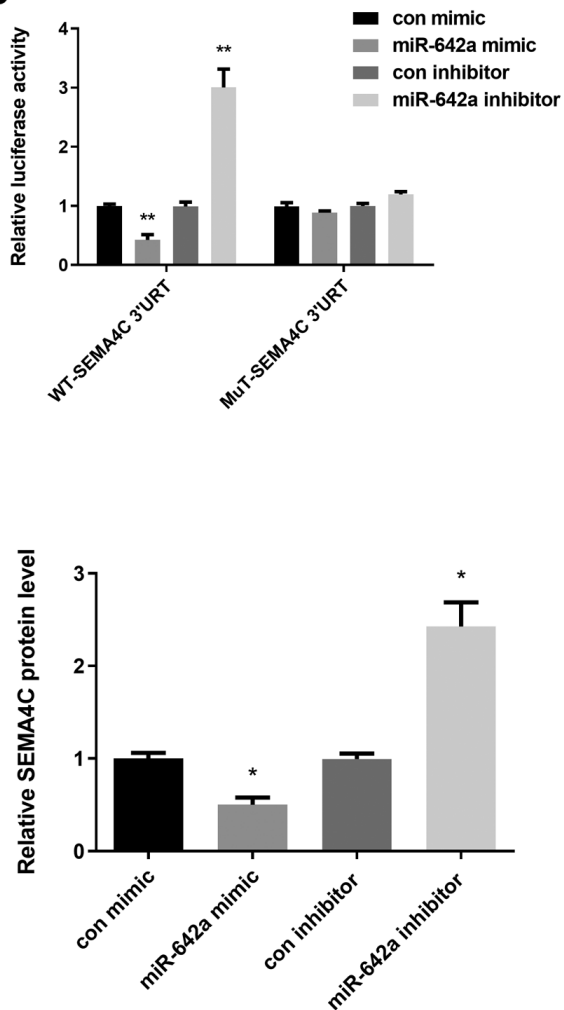

Figure 2. SEMA4C is a target of miR-642a. (A) Prediction of the binding sites of miR-642a and SEMA4C. (B) Luciferase activity of SEMA4C-3'UTR-WT or SEMA4C-3'UTR-MuT was detected in Huh7 cells after transfected with miR-642a mimic or inhibitor. (C) Comparison of SEMA4C expression in Huh7 cells by western blot analysis and (D) RT-PCR after treated with miR-642a mimic or miR-642a inhibitor. ${ }^{*} \mathrm{P}<0.05,{ }^{* *} \mathrm{P}<0.01$.

miR-642a mimic or inhibitor was transfected into Huh7 cells to measure the effect of miR-642a on HCC cell migration and invasion. As shown in Fig. 3A, the transfection efficiency of miR-642a was successful. The expression of miR-642a was increased significantly in Huh7 cells after overexpression of miR-642a, while decreased after knockdown of miR-642a. Then, Transwell assays were applied to examine HCC cell migration and invasion. As presented in Fig. 3B, the number of migrated cells declined significantly in miR-642a mimic group, whereas were elevated in miR-642a inhibitor group. Moreover, the invasive cells of Huh7 were decreased after re-expression of miR-642a, while increased after inhibiting miR-642a (Fig. 3C).

Silencing SEMA4C suppresses HCC malignancy. Next, the effects of SEMA4C on HCC cell migration and invasion were detected. Due to the higher expression of SEMA4C in HCC, it was decreased by SEMA4C siRNA. As expected,
SEMA4C expression was declined remarkably after silencing SEMA4C in Huh7 cells (Fig. 4A). Transwell assay was then applied to measure the migration and invasion of Huh7 cells affected by SEMA4C siRNA. As presented in Fig. 4B, SEMA4C siRNA suppressed Huh7 cell migration. Moreover, silencing SEMA4C repressed Huh7 cell invasion (Fig. 4C).

The effect of SEMA4C on miR-642 in the modulation of HCC malignancy. The effect of SEMA4C on miR-642a in regulating HCC malignancy was explored due to their opposite effect on HCC invasion and migration. Huh7 cells were treated with different transfections: con inhibitor+con siRNA, miR-642a inhibitor+con siRNA, miR-642a inhibitor+SEMA4C siRNA. Transwell migration assay results displayed that the migration of Huh7 cells was increased obviously by silencing miR-642a, while decreased by silencing miR-642 and SEMA4C, suggesting that SEMA4C could rescue miR-642a suppression 
A

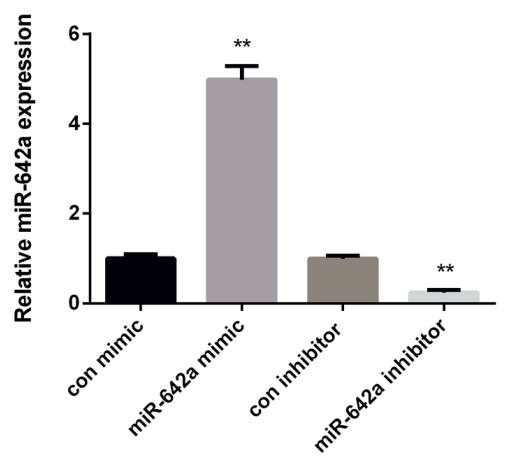

B
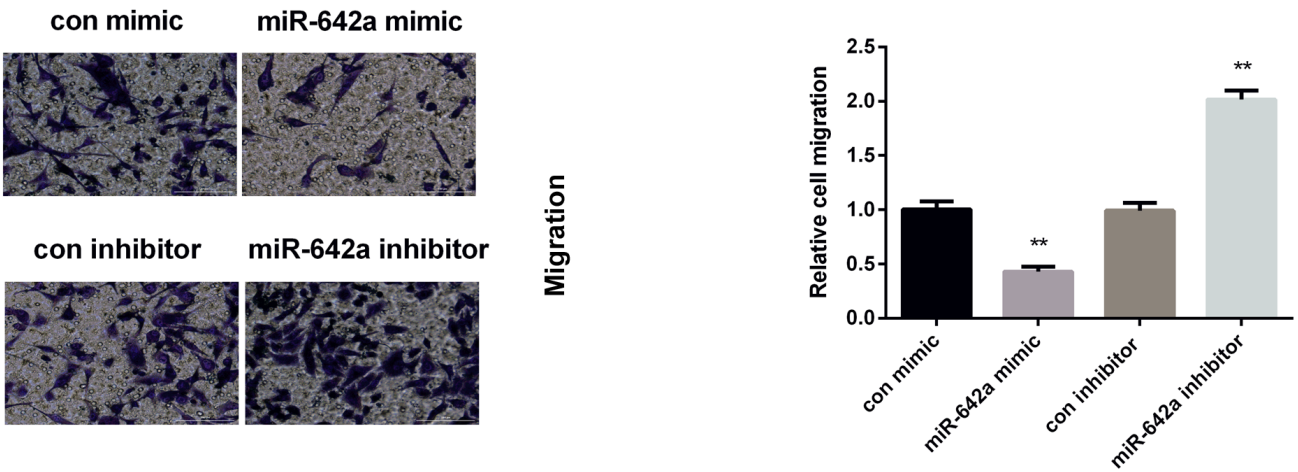

C
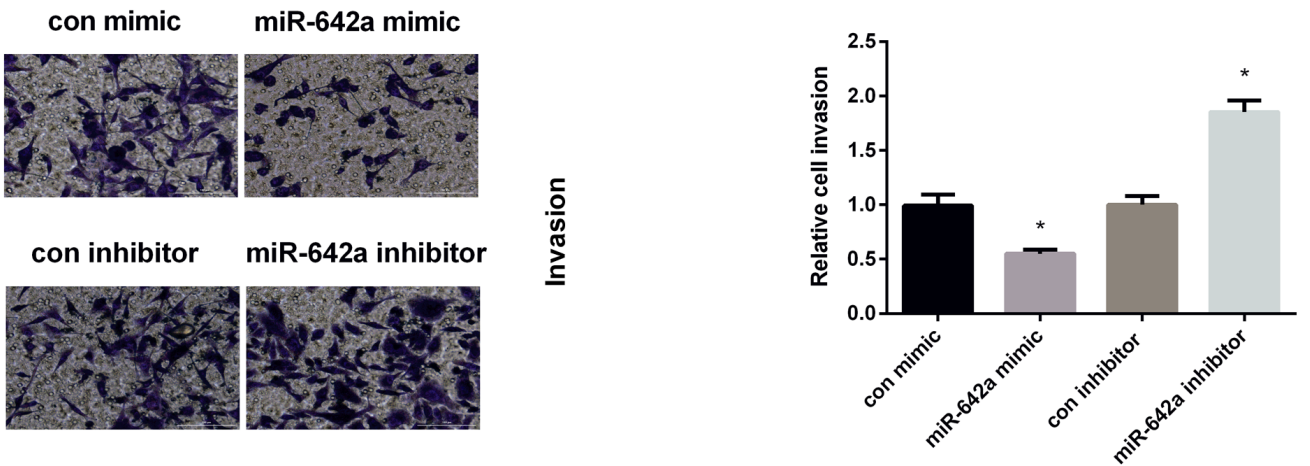

Figure 3. miR-642a suppresses HCC cell invasion and migration. (A) Measurement of miR-642a expression in Huh7 cells after overexpression or knockdown of miR-642a. (B) Comparison of cell migration in Huh7 cells after treated with miR-642a mimic or inhibitor. (C) Comparison of cell invasion in Huh7 cells after treated with miR-642a mimic or inhibitor. ${ }^{*} \mathrm{P}<0.05,{ }^{* *} \mathrm{P}<0.01$. HCC, hepatocellular carcinoma.

effect on cell migration (Fig. 5A). Moreover, the results of Transwell invasion assay demonstrated that downregulation of miR-642a increased HCC cell invasion, whereas was reduced by downregulation of miR-642a and SEMA4C, indicating that SEMA4C could overturn the inhibition effect of miR-642a on HCC cell invasion (Fig. 5B). Correctively, miR-642a curbed the invasion and migration of HCC cells by modulating SEMA4C.

p38 MAPK signaling pathway is involved in HCC development regulated by miR-642a/SEMA4C axis. Finally, we investigated whether p38 MAPK signaling pathway was modulated by miR-642a/SEMA4C axis to explore the precise molecular mechanism of miR-642a in the development of HCC. Results of Western blot analysis displayed that the downstream genes of p38 MAPK pathway were inhibited by miR-642a upregulation, while enhanced by miR-642a inhibitor. Moreover, silenced SEMA4C was able to repress the activation of phosphorylation of $\mathrm{p} 38$ and MAPK induced by miR-642a inhibitor (Fig. 6). These results demonstrated that miR-642a impeded the activation of $\mathrm{p} 38$ MAPK signaling pathway by suppressing SEMA4C in HCC.

\section{Discussion}

Our findings displayed that miR-642a expression was declined while SEMA4C was raised in HCC tissues and cells. Moreover, miR-642a showed inhibitory effect on HCC malignancy and SEMA4C showed the opposite effect. Importantly, SEMA4C was proved to be the target 
A

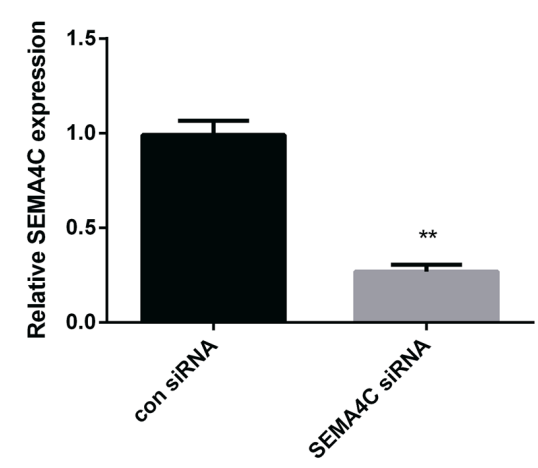

B
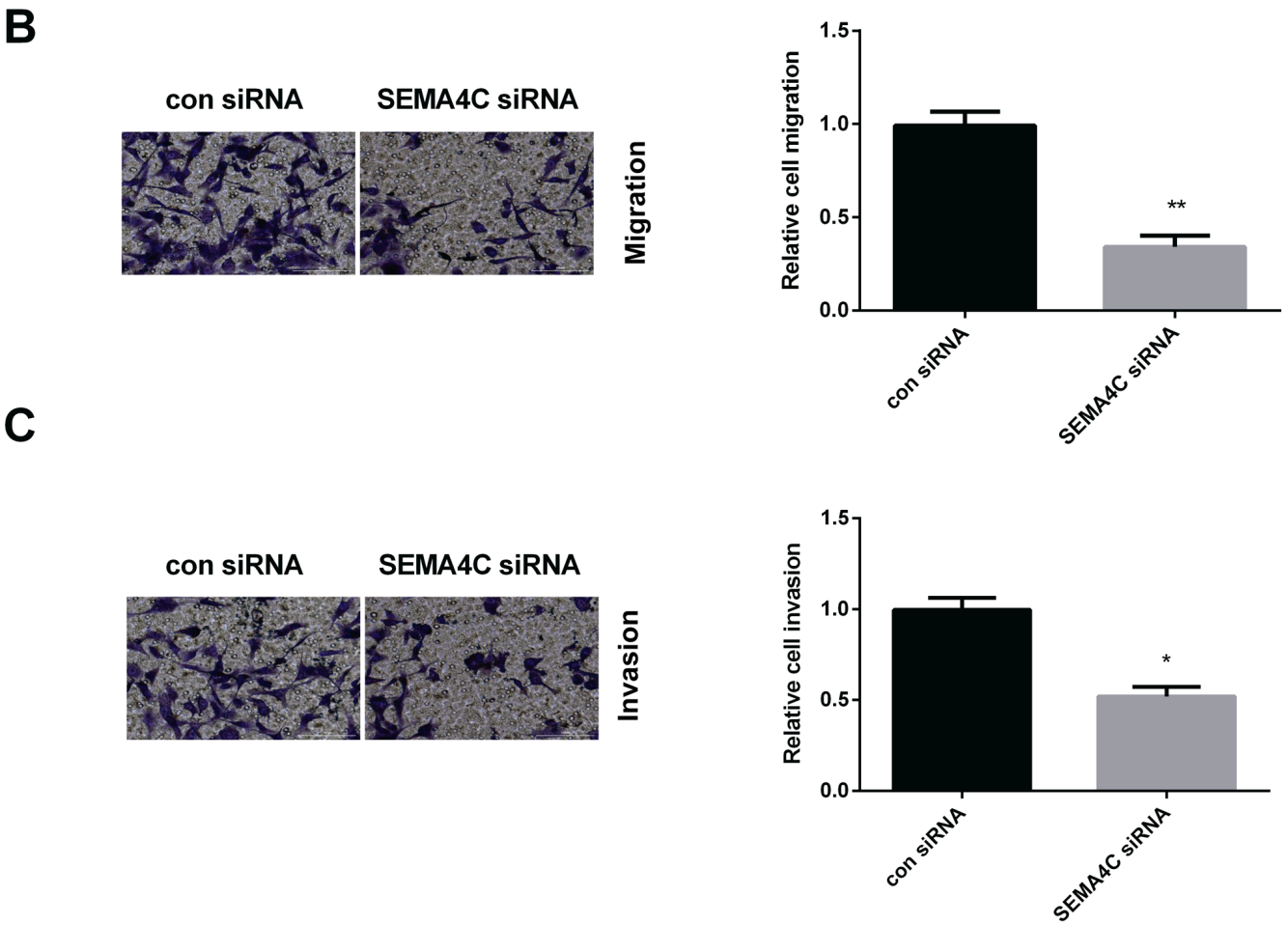

Figure 4. SEMA4C siRNA inhibits HCC cell invasion and migration. (A) Detection of SEMA4C expression in Huh7 cells after silencing SEMA4C by RT-PCR and western blot analysis. (B) Comparison of cell migration in Huh7 cells after treated with SEMA4C siRNA and con siRNA. (C) Comparison of cell invasion in Huh7 cells after treated with SEMA4C siRNA or con siRNA. ${ }^{*} \mathrm{P}<0.05,{ }^{* *} \mathrm{P}<0.01$. HCC, hepatocellular carcinoma.

of miR-642a in regulation of HCC development. Moreover, SEMA4C could rescue the inhibitory effect of miR-642a on HCC malignancy. In addition, p38 MAPK signaling pathway was modulated by miR-642a/SEMA4C axis. Taken together, the research demonstrated that miR-642a inhibited HCC development by repressing SEMA4C through p38 MAPK signaling pathway, providing a potential target for treatment of HCC patients.

Increasing evidence has been reported that miRNAs modulated various molecular pathways in tumor development via targeting their specific mRNAs as either tumor suppressors or oncogenes (18-20). Previous studies stated that miRNA expression profiles varied from cancer to cancer. For example, miR-642a was involved in pancreatic neuroendocrine neoplasms and correlated with Ki67 score (21). Moreover, Nordentoft et al (22) revealed that increasing miR-642 generally increased cisplatin sensitivity of bladder cancer and might form a novel target for treatment of patients.
However, Epis et al (23) showed an under-expression of miR-642 in prostate cancer and overexpression of miR-642 suppressed cell proliferation. In the present study, it was found that miR-642a was under-expressed in HCC tissues and cells which is consistent with the report that miR-642a expression was downregulated in HCC and took part in HCC proliferation and metastasis regulated by Linc00974 and KRT19 (12). In this study, we also found that re-expression of miR-642a repressed $\mathrm{HCC}$ invasion and migration.

SEMA4C was reported highly expressed in numerous cancer tissues, such as in gastric cancer, esophageal cancer and rectal cancer (24). SEMA4C usually take part in tumor development as a target of miRNA. For instance, it was a target of miR-25 in regulating the epithelial-mesenchymal transition of cervical cancer (25). Also, SEMA4C was regulated by miR-125b in regulating epithelial-mesenchymal transition of lung cancer (26). Li et al (27) stated that miR-138 suppressed non-small cell lung cancer cell proliferation through targeting 
A
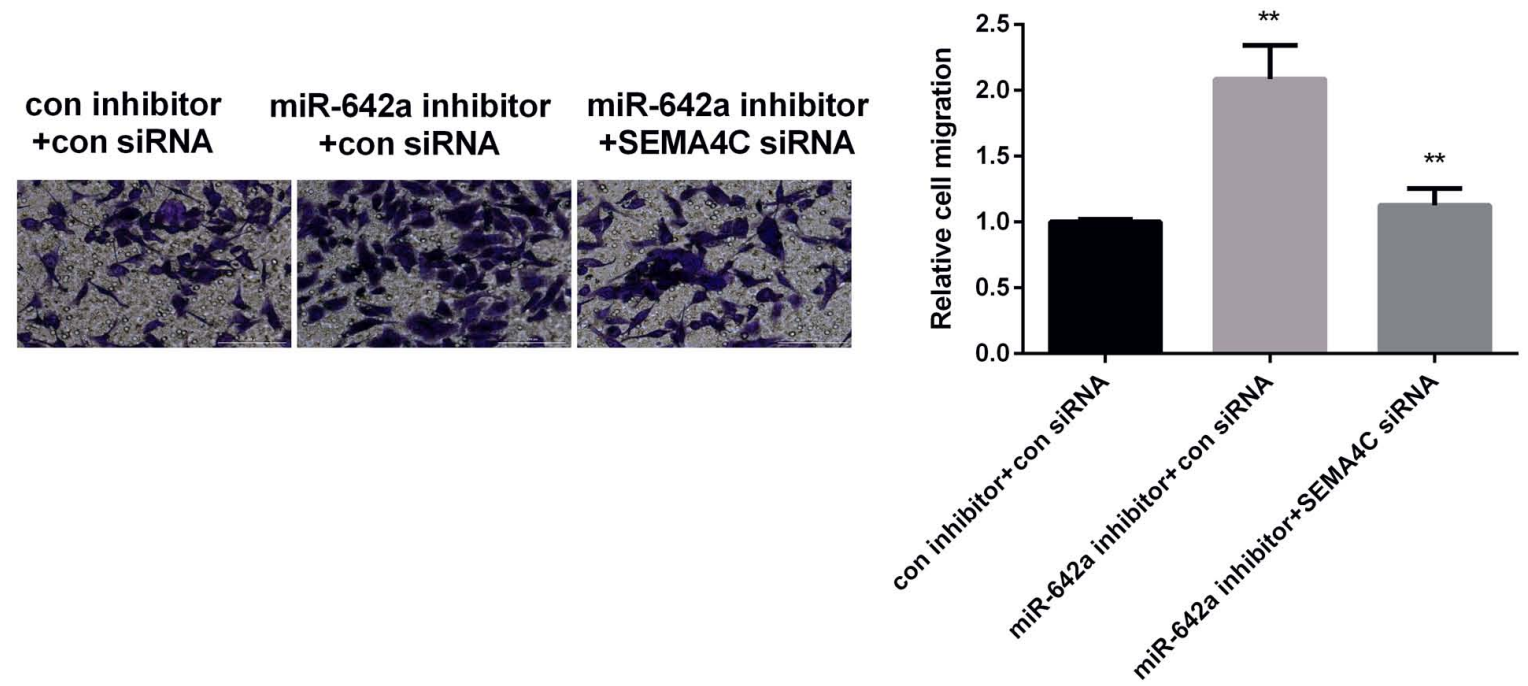

B
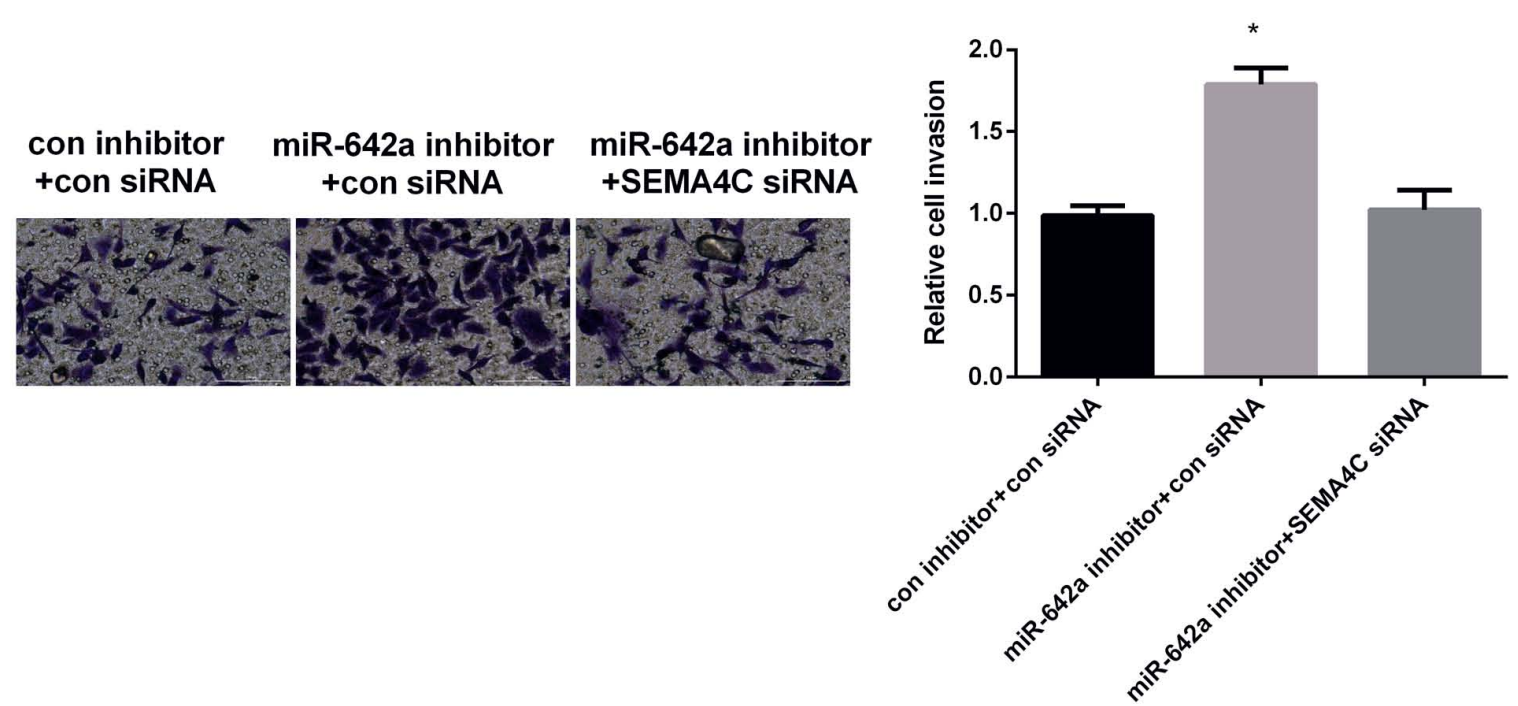

Figure 5. SEMA4C reverses miR-642a inhibitory effect on HCC cell migration and invasion. (A) Comparison of Huh7 cell migration in miR-642a inhibitor group or combined with SEMA4C siRNA group. (B) Comparison of Huh7 cell invasion in miR-642a inhibitor group or combined with SEMA4C siRNA group. ${ }^{*} \mathrm{P}<0.05,{ }^{* *} \mathrm{P}<0.01$. HCC, hepatocellular carcinoma.

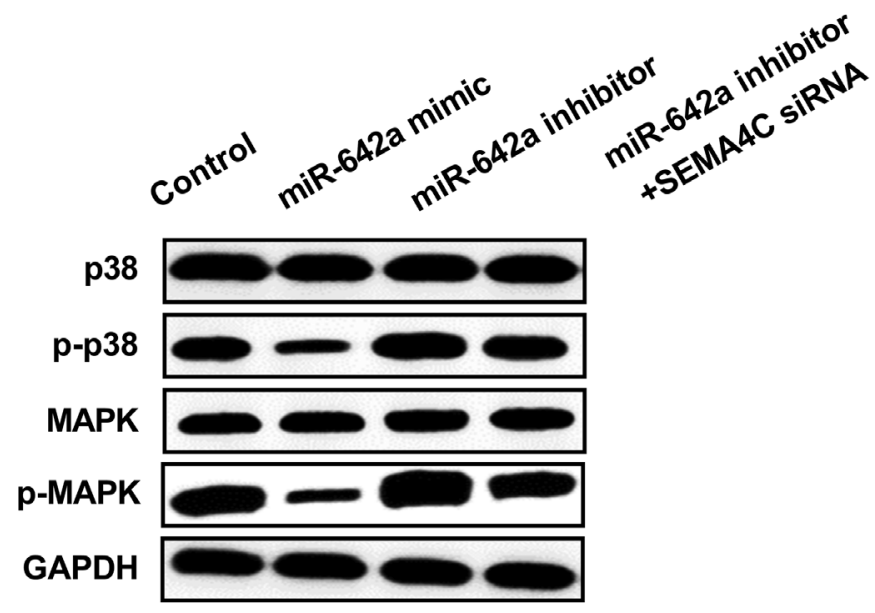

Figure 6. miR-642a represses p38 MAPK signaling pathway via targeting SEMA4C. Western blot analysis of p-p38, p38, p-MAPK and MAPK levels in Huh7 cells after treated with miR-642a mimic, inhibitor or combined with SEMA4C siRNA.
GIT1 and SEMA4C. In our study, the SEMA4C expression was raised in HCC and silencing SEMA4C was able to curb HCC malignancy. These results are in line with a previous study that SEMA4C was overexpressed in HCC and involved in HCC tumor growth and invasion (16). Furthermore, we first found that SEMA4C was a specific target of miR-642a in the regulation of HCC malignancy and SEMA4C could rescue miR-642a inhibitory effect on HCC malignancy.

In the tumor development and metastasis, p38 MAPK signaling pathway is activated as a frequent event $(28,29)$. p38 MAPK pathway was dysregulated in HCC tissues compared with the corresponding normal tissues (30). Increasing number of studies have demonstrated that miRNAs interacted with signaling pathways in a variety of cancers. In the present study, we found that p38 MAPK signaling pathway was inhibited by miR-642a mimic, whereas activated by miR-642a inhibitor. Furthermore, SEMA4C siRNA attenuated the activation of p38 MAPK signaling pathway induced by miR-642a inhibitor. 
There are limitations in the present study. The role of the miR-642a/SEMA4C axis should be verified in vivo. The effect of SEMA4C overexpression or knockdown on p38 MAPK signaling pathway remain to be done, which will guide the investigation into the inmost mechanism. The association of miR-642a with clinical characteristics of patients should be done in future studies.

In conclusion, our findings revealed that miR-642a suppressed, while SEMA4C promoted HCC malignancy. This is the first time we state that miR-642a directly targeted SEMA4C to modulate HCC development and SEMA4C could overturn miR-642a inhibitory effect on HCC. Taken together, by targeting SEMA4C, miR-642a impeded HCC malignancy through suppressing p38 MAPK signaling pathway.

\section{Acknowledgements}

Not applicable.

\section{Funding}

The study was supported by the Fujian Natural Science Foundation (Fujian, China) (grant no. 2019J01188).

\section{Availability of data and materials}

The datasets used and analyzed during the present study are available from the corresponding author on reasonable request.

\section{Authors' contributions}

QK contributed to the conception and design of the study. HL and $\mathrm{JH}$ analyzed and interpreted the patient general data. DJ, JF, YS, LZ, XY and NX performed RT-PCR, Transwell assay, western blot analysis and luciferase reporter assay. ZY and YD were also involved in the conception of the study. QK gave final approval of the version to be published. All authors read and approved the final version of the manuscript.

\section{Ethics approval and consent to participate}

The study was approved by the Ethics Committee of The Second People's Hospital of Lianyungang (Lianyungang, China) and an informed consent was signed by each patient.

\section{Patient consent for publication}

Not applicable.

\section{Competing interests}

The authors declare that they have no competing interests.

\section{References}

1. Siegel RL, Miller KD and Jemal A: Cancer Statistics, 2017. CA Cancer J Clin 67: 7-30, 2017.

2. Fornari F, Ferracin M, Trerè D, Milazzo M, Marinelli S, Galassi M, Venerandi L, Pollutri D, Patrizi C, Borghi A, et al: Circulating microRNAs, miR-939, miR-595, miR-519d and miR-494, identify cirrhotic patients with HCC. PLoS One 10: e0141448, 2015.
3. Bray F, Ferlay J, Soerjomataram I, Siegel RL, Torre LA and Jemal A: Global cancer statistics 2018: GLOBOCAN estimates of incidence and mortality worldwide for 36 cancers in 185 countries. CA Cancer J Clin 68: 394-424, 2018.

4. Kassahun WT, Fangmann J, Harms J, Hauss J and Bartels M: Liver resection and transplantation in the management of hepatocellular carcinoma: A review. Exp Clin Transplant 4: 549-558, 2006.

5. Dilou N, Patouillard B and Audigier JC: Staging systems in hepatocellular carcinoma. Gastroenterol Clin Biol 28: 359-366, 2004 (In French).

6. Ono K, Kuwabara Y and Han J: MicroRNAs and cardiovascular diseases. FEBS J 278: 1619-1633, 2011.

7. Lim LP, Lau NC, Garrett-Engele P, Grimson A, Schelter JM, Castle J, Bartel DP, Linsley PS and Johnson JM: Microarray analysis shows that some microRNAs downregulate large numbers of target mRNAs. Nature 433: 769-773, 2005.

8. Zhao S, Li J, Zhang G, Wang Q, Wu C, Zhang Q, Wang H, Sun P, Xiang R and Yang S: Exosomal miR-451a functions as a tumor suppressor in hepatocellular carcinoma by targeting LPIN1. Cell Physiol Biochem 53: 19-35, 2019.

9. Wu J, Huang WJ, Xi HL, Liu LY, Wang ST, Fan WZ and Peng BG: Tumor-suppressive miR-3650 inhibits tumor metastasis by directly targeting NFASC in hepatocellular carcinoma. Aging (Albany NY) 11: 3432-3434, 2019.

10. Chen S, Wang L, Yao B, Liu Q and Guo C: miR-1307-3p promotes tumor growth and metastasis of hepatocellular carcinoma by repressing DAB2 interacting protein. Biomed Pharmacother 117: 109055,2019

11. Zhang J, Zhu Y, Hu L, Yan F and Chen J: miR-494 induces EndMT and promotes the development of HCC (hepatocellular carcinoma) by targeting SIRT3/TGF- $\beta /$ SMAD signaling pathway. Sci Rep 9: 7213, 2019.

12. Tang J, Zhuo H, Zhang X, Jiang R, Ji J, Deng L, Qian X, Zhang F and Sun B: A novel biomarker Linc00974 interacting with KRT19 promotes proliferation and metastasis in hepatocellular carcinoma. Cell Death Dis 5: e1549, 2014.

13. Gurrapu S, Pupo E, Franzolin G, Lanzetti L and Tamagnone L: Sema4C/PlexinB2 signaling controls breast cancer cell growth, hormonal dependence and tumorigenic potential. Cell Death Differ 25: 1259-1275, 2018.

14. Wei JC, Yang J, Liu D, Wu MF, Qiao L, Wang JN, Ma QF, Zeng Z, Ye SM, Guo ES, et al: Tumor-associated lymphatic endothelial cells promote lymphatic metastasis by highly expressing and secreting SEMA4C. Clin Cancer Res 23: 214-224, 2017.

15. Le AP, Huang Y, Pingle SC, Kesari S, Wang H, Yong RL, Zou $\mathrm{H}$ and Friedel RH: Plexin-B2 promotes invasive growth of malignant glioma. Oncotarget 6: 7293-7304, 2015.

16. Lu J, Lin Y, Li F, Ye H, Zhou R, Jin Y, Li B, Xiong X and Cheng N: miR-205 suppresses tumor growth, invasion, and epithelial-mesenchymal transition by targeting SEMA4C in hepatocellular carcinoma. FASEB J 32: fj201800113R, 2018.

17. Wu H, Wang X, Liu S, Wu Y, Zhao T, Chen X, Zhu L, Wu Y, Ding X, Peng X, et al: Sema4C participates in myogenic differentiation in vivo and in vitro through the p38 MAPK pathway. Eur J Cell Biol 86: 331-344, 2007.

18. Wang ZF, Liao F, Wu H and Dai J: Glioma stem cell-derived exosomal miR-26a promotes angiogenesis of microvessel endothelial cells in glioma. J Exp Clin Cancer Res 38: 201, 2019.

19. Zhang J, Hou L, Liang R, Chen X, Zhang R, Chen W and Zhu J: CircDLST promotes the tumorigenesis and metastasis of gastric cancer by sponging miR-502-5p and activating the NRAS/MEK1/ERK1/2 signaling. Mol Cancer 18: 80, 2019.

20. Ma M, Dai J, Tang H, Xu T, Yu S, Si L, Cui C, Sheng X, Chi Z, MaoL, et al: MicroRNA-23a-3p inhibits mucosal melanoma growth and progression through targeting adenylate cyclase 1 and attenuating cAMP and MAPK pathways. Theranostics 9: 945-960, 2019.

21. Thorns C, Schurmann C, Gebauer N, Wallaschofski H, Kümpers C, Bernard V, Feller AC, Keck T, Habermann JK, Begum N, et al: Global microRNA profiling of pancreatic neuroendocrine neoplasias. Anticancer Res 34: 2249-2254, 2014.

22. Nordentoft I, Birkenkamp-Demtroder K, Agerbæk M, Theodorescu D, Ostenfeld MS, Hartmann A, Borre M, Ørntoft TF and Dyrskjøt L: miRNAs associated with chemo-sensitivity in cell lines and in advanced bladder cancer. BMC Med Genomics 5: 40, 2012.

23. Epis MR, Giles KM, Kalinowski FC, Barker A, Cohen RJ and Leedman PJ: Regulation of expression of deoxyhypusine hydroxylase $(\mathrm{DOHH})$, the enzyme that catalyzes the activation of eIF5A, by miR-331-3p and miR-642-5p in prostate cancer cells. J Biol Chem 287: 35251-35259, 2012. 
24. Ye SM, Han M, Kan CY, Yang LL, Yang J, Ma QF and Wang SX Expression and clinical significance of Sema4C in esophageal cancer, gastric cancer and rectal cancer. Zhonghua Yi Xue Za Zhi 92: 1954-1958, 2012 (In Chinese).

25. Song J and Li Y: miR-25-3p reverses epithelial-mesenchymal transition via targeting Sema4C in cisplatin-resistance cervical cancer cells. Cancer Sci 108: 23-31, 2017.

26. Zhang Y and Huang S: Up-regulation of miR-125b reverses epithelial-mesenchymal transition in paclitaxel-resistant lung cancer cells. Biol Chem: Aug 20, 2015 (Epub ahead of print). doi: 10.1515/hsz-2015-0153.

27. Li J, Wang Q, Wen R, Liang J, Zhong X, Yang W, Su D and Tang J: miR-138 inhibits cell proliferation and reverses epithelial-mesenchymal transition in non-small cell lung cancer cells by targeting GIT1 and SEMA4C. J Cell Mol Med 19: 2793-2805, 2015.

28. Chen J, Ji T, Wu D, Jiang S, Zhao J, Lin H and Cai X: Human mesenchymal stem cells promote tumor growth via MAPK pathway and metastasis by epithelial mesenchymal transition and integrin $\alpha 5$ in hepatocellular carcinoma. Cell Death Dis 10 : $425,2019$.
29. Chatterjee S, Patra D, Chakraborti U, Sengupta D, Ghosh P, Basu A, Sadhukhan GC and Chowdhury KD: Association of p38 MAPK-p53-Fas aggregation in S-allyl cysteine mediated regulation of hepatocarcinoma. Environ Toxicol 34: 928-940, 2019.

30. Zhang GP, Yue X and Li SQ: Cathepsin C interacts with TNF-alpha/p38 MAPK signaling pathway to promote proliferation and metastasis in hepatocellular carcinoma. Cancer Res Treat 52: 10-23, 2020.

(i)(9) This work is licensed under a Creative Commons Attribution-NonCommercial-NoDerivatives 4.0 International (CC BY-NC-ND 4.0) License. 